\title{
The coordinated development of Beijing-Tianjing-Hebei urban research
}

\author{
Zheng Yongwu \\ Graduate School Of Henan University, Henan, Kaifeng \\ Zhang chongcong \\ Hebei Normal University Business School, \\ Hebei province, shijiazhuang \\ Zhang jian \\ Pennsylvania State University, The United States
}

\begin{abstract}
The Beijing-Tianjing-Hebei in China plays a decisive role in economic and social development. The coordinated development of urbanization of the Beijing-TianjingHebei region, will greatly improve the level of regional urbanization, promote regional economic development, promote urbanization and economic development of Bohai rim region. Adhere to the principle of complementary advantages and win-win cooperation, establish the coordinated development of Beijing-Tianjing-Hebei urban mechanism, overall planning of Beijing-Tianjing-Hebei urbanization. In order to improve the urbanization level as the goal of the Beijing-Tianjing-Hebei region, optimization of regional industry layout, strengthen ecological environment comprehensive treatment, and strengthening regional urban construction coordination, coordination and ecological environment protection coordination of industrial development, realize the coordinated development of Beijing-Tianjing-Hebei urbanization.
\end{abstract}

Key words: Beijing-Tianjing-Hebei; Urbanization; Synergy; The development of

Xi Jinping pointed out: Beijing, Tianjing and Hebei added up to more than 100 million population, land area of 21.6 square kilometers, Beijing-Tianjing-Hebei geopolitical connect, dating popular, regional, cultural, historical origin, deep communication radius and BeijingTianjing-Hebei is fully capable of mutual confluence, collaborative development. To promote the coordinated development of urbanization of the Beijing-Tianjing-Hebei region, should be based on their respective comparative advantage, based on the requirements of modern industrial division of labor and based on the regional advantage complementary principle, based on the concept of win-win cooperation, according to the construction of Beijing-TianjingHebei urban agglomeration as the carrier, focusing on the optimization of regional division of labor and industry layout, with overall planning elements of space utilization of resources as the main line, in order to build the system mechanism for the gripper, speed up the development of [1] from the breadth and depth.

\section{THE MEANING OF BEIJING-TIANJING-HEBEI URBAN COORDINATED DEVELOPMENT}

Xi stressed: realize the coordinated development of Beijing-Tianjing-Hebei, is for the future to build a new capital economic circle, the need of system and mechanism innovation, promote regional development is to explore perfect urban layout and form, in order to optimize the development of regional development provides the demonstration and the needs of the 
template, is to explore an effective way for construction of ecological civilization, and promote the population, the needs of economic resources and environment in harmony, is to realize the complementary advantages of the Beijing-Tianjing-Hebei region, promote the development of the Bohai sea economic zone, to drive the needs of the development of the hinterland, is a major national strategy, insist on the complementary advantages, mutual benefit and win-win results, made solid progress, speed up out of the ongoing collaborative development with a scientific to [2].

\section{Is beneficial to realize national development strategy}

Beijing-Tianjing-Hebei region is the political center and cultural center of China, north China economic center. Because of the influence of the administrative division, Beijing-TianjingHebei fragmented in three provinces and cities in the region urbanization development, seriously restricted the rapid development of the region economy, affected the overall regional interests and competitiveness. Implementation of the coordinated development of urbanization of the Beijing-Tianjing-Hebei region, is not only beneficial to improve the level of regional urbanization, promote regional economic development, but also to promote urbanization and economic development, the Bohai rim region is beneficial to realize national development strategy.

\section{To promote healthy development of the Beijing and Tianjing two cities}

Beijing-Tianjing-Hebei region of the two cities of Beijing, Tianjing and the surrounding areas on the urban development disjointed, self-closed, fragmented, core cities on the surrounding radiation function not well. The Beijing and Tianjing two city is China's most abundant education resources, talent resources, science and technology resources, these resources are not give full play to the function of. In Hebei province as the coordinated development of urbanization in the periphery of the Beijing-Tianjing-Hebei region, is the Beijing and Tianjing energy and raw materials supply base and the important channel, is of great importance in the Beijing and Tianjing technology, talent and commodity markets, is an important ecological barrier of the Beijing and Tianjing and water supply. Implementation of the coordinated development of urbanization of the Beijing-Tianjing-Hebei region, to improve the Beijing and Tianjing, urbanization is beneficial to greatly improve the quality of the Beijing-Tianjing urbanization, is beneficial to optimizing the Beijing-Tianjing industrial layout, is beneficial to improve the regional ecological environment, promote the Beijing and Tianjing's economic and social healthy development.

\section{To promote regional economic and social development}

Beijing-Tianjing-Hebei collaborative development strategy is put forward, bring great opportunities for the coordinated development of Beijing-Tianjing-Hebei urban. As a whole of the Beijing-Tianjing-Hebei region, three different provinces and cities development is bound to affect the regional development scale and speed of development. Beijing and Tianjing is the center of the coordinated development of urbanization of the Beijing-Tianjing-Hebei region, is the core of the Beijing-Tianjing-Hebei economic development area, Tianjing's development inevitably affects the development of the Beijing-Tianjing-Hebei region. Any one area of development is inseparable from the adjacent areas of development, the development of Beijing and Tianjing will inevitably affect the economic development in Hebei province, Hebei province development also affects the development of Beijing and Tianjing. To speed up the urbanization coordinated development of the Beijing-Tianjing-Hebei region, Beijing-TianjingHebei urban development planning as a whole, is not only beneficial to promote the 
development of urbanization in Hebei, and conducive to promoting urbanization, the Beijing and Tianjing is conducive to promoting regional economic and social development.

\section{SECOND, THE BEIJING-TIANJING-HEBEI URBAN PROBLEMS \\ Regional urbanization level gap is big The Beijing and Tianjing central city development deformity}

Consciousness as a regional nuclear two cities of Beijing, Tianjing and the surrounding areas of relatively independent in the development of urban agglomerations disjointed, self-closed, fragmented, loosely connected each other space, around the core city of urban economic radiation function.

At the end of 2013 the total population of more than 109 million people of the Beijing-TianjingHebei region. In 2012, a total population of 20.69 million people in Beijing, Tianjing in 2012, a total population of 14.13 million people [3]. From the point of population flows, both Beijing and Tianjing is the largest area of cross-regional population inflows. Close to the population of permanent residents in the migrant population in Beijing at the end of 2013, 8 million, an annual increase of resident population of more than 80; Tianjing since 2008 to keep the average annual increment of about 600000 .

Beijing and Tianjing economic development, employment, income level is high, become the important factor of attracting migrants. In the outer ring of Beijing-Tianjing, Hebei province, because of the distance to Beijing and Tianjing, running cost is relatively low. Because the Beijing-Tianjing-Hebei long-term in a region, the floating population were also more likely to get the psychological identity. According to the sixth census data in 2010, the 2010 census point to a population of 3.498 million people outside the province, Hebei province, including flow to Beijing's population of 1.559 million people, accounting for $44.6 \%$ of the population outflow. Flow to the Tianjing a population of 755000 people, accounting for $21.6 \%$ of the population outflow, flow to the Beijing and Tianjing population account for $66.2 \%$ of the outflow of population in Hebei province [4].

From 2001 to 2012, the population growth in the Beijing-Tianjing-Hebei region presents two characteristics: one is the population is growing rapidly, Beijing-Tianjing-Hebei population reached 107.7 million in 2012, compared with 2001 growth of 16.82 million people, an increase of $1.19 \%$. Second, the imbalance of population growth. Beijing population reached 20.69 million in 2012, compared with 2001 growth of 6.84 million people, an increase of $1.49 \%$. Between 2001 and 2012, Beijing's population growth is more than $0.4 \%$ of the BeijingTianjing-Hebei overall growth. Tianjing population reached 14.13 million in 2012, compared with 2001 growth of 2001 people, an increase of 1.41\%. From 2001 to 2012 in Tianjing, population growth is more than $0.32 \%$ of Beijing-Tianjing-Hebei overall growth [5]. Total population reached 72.88 million in 2012, Hebei province, compared with 2001 growth of 2001 people, an increase of $1.09 \%$. From 2001 to 2012 in Hebei province population growth below the Beijing-Tianjing-Hebei overall increase of $0.4 \%$. See the table below.

The Beijing and Tianjing area in the middle of industrialization and urbanization fast development, the future will be quite a long period of time the population continued to flow region. Excessive resources and financial resources to Beijing city center, leading to Beijing "big city disease" is more and more serious, the capital has become the first wall. Tianjing, the second largest city in the Beijing-Tianjing-Hebei region, due to urban growth too fast, cause disease of big cities is very serious, serious influence the exertion of urban functions. 


\section{The center city development lags behind}

Beijing-Tianjing-Hebei regional urban system development imbalances, secondary center city development lags behind. Beijing-Tianjing-Hebei region in addition to the city center of Beijing population size more than 20 million, Tianjing downtown more than 14 million, other districts, urban population scale only in shijiazhuang and tangshan city has a population of more than 3 million. From 2000 to 2010, the average annual growth rates of shijiazhuang, tangshan resident population less than one over ten of Beijing. Lack of urban size, economic radiation ability is not strong.

2010 population between 1 million $\sim 2$ million is handan city in Hebei province, baoding, zhangjiakou. Handan city governs 4 area 1 in 14 counties, a total area of 12000 square kilometers, jurisdiction of baoding city 3 area 4 city 18 county, with a total area of 22100 square kilometers. Governs 4 zhangjiakou district 13 county with a total area of 36860 square kilometers. Handan city urban population reached 1.41 million in 2010, baoding urban population of 1.11 million people, the city's population reached 1.02 million zhangjiakou, three cities population account for $27.4 \%$ of the population of the city of Hebei province [6]. City characteristic economy and pillar industry development is not prominent, the industrial structure identical, division of labor cooperation and coordination of sharing pattern has not yet formed.

Population of 1 million in Hebei province in 2010 the following six in the center of the city, qinhuangdao comprises 3 area 4 counties, a total area of 7812 square kilometers, the urban population of 990000 . Langfang area 2 cities of 8 counties with a total area of 6500 square kilometers, the urban population of 870000 . Xingtai comprises 2 area 2 city 15 counties, a total area of 12486 square kilometers, the urban population of 740000. Cangzhou area 2 and 4 cities of 10 counties, a total area of 13419 square kilometers, the urban population of 620000 . Chengde city comprises three district 8 counties with a total area of 39519 square kilometers, the urban population of 590000. The urban population is the least amount of hengshui city, hengshui city comprises 1 area 2, 8 counties, a total area of 8815 square kilometers, the urban population of 460000 .

Time central cities in Hebei province population scale, comprehensive strength, urban function, innovation ability and the urban competitiveness, compared with the Beijing and Tianjing city exists great gap, as big as Yangtze river delta, the pearl river delta is also very obvious gap compared to the city.

\section{Shortage of regional small and medium-sized city function}

Is a medium-sized city function is not strong. Area county in Hebei province city function is insufficient, county economic development is relatively slow, not strong in the population. Medium-sized cities of regional economic development has a crucial role, which forms a connecting link between the preceding and medium-sized city in Hebei province city function is generally weak, attract population capacity is not strong, lost momentum of economic development, the essential function is weak, difficult to drive the area economic development. In Hebei province in 2012 to more than 140 counties (cities) reached more than 6000 population, $60 \%$ of the total population of Beijing-Tianjing-Hebei region, and county GDP, total retail sales of social consumer accounts for only a third of the Beijing-Tianjing-Hebei region, the added value of the tertiary industry accounted for a quarter of the Beijing-Tianjing-Hebei region [7]. 
Second, the small towns development of poor quality, a serious shortage of urban functions. Small towns in Hebei province showed the following characteristics: one is that most of the small town is too small, difficult to form scale; The second is the low level of urbanization of small towns in Hebei province, most of the urban infrastructure is not sound, the urbanization level is not high, the city function is not complete. Three small towns in Hebei province is strength is not strong, the various elements gathering force is weak, the economic development function is weak in [8].

\section{The administrative division Whose urban planning}

The development of regional economy depends on the establishment of regional coordinated development mechanism and effective operation. For a long time, the administrative division of the Beijing-Tianjing-Hebei region is very serious, fragmented urban planning, the function of market mechanism to adjust the economy cannot be fully effectively, the regional economic efficiency by a lot of harm. The administrative subject to being lack of cooperation and regional development of major strategic space in major industry layout, key resources development and major infrastructure construction, important aspects such as lack of overall consideration and coordination of regional ecological protection, [9] seriously weakened the regional economic development advantage.

\section{The resources advantage of competition}

Driven by the interests of the administrative subject in departments at various levels, regardless of the effect of external blind area, the development and construction main body from their respective interests, not considering the influence of the development of ecological environment and excessive use of resources, from the regional ecological space, greatly harm the interests of the regional overall development. Independent of the Beijing-Tianjing-Hebei region logistics infrastructure, logistics facilities using competition, the organization of logistics lack of negotiation and cooperation. A lack of continuity between Beijing-Tianjing-Hebei regional transportation network, city is still a long commuting distance and time; Regional integrated transport network less foreign channels, main ability seriously insufficient; Regional integrated transport network in any mode of transport do not coordinate each other, do not match, cohesion, transportation time is long, transit times, ports, railways, highways set hydrophobic ability is insufficient. Repetition of port construction, serious waste of resources. [10]

\section{Use less than factors of urban development}

From the point of urbanization elements configuration, the Beijing and Tianjing are the two cities in Hebei province rural labor on major areas of employment, the Beijing and Tianjing two cities lack effective coordination mechanism in terms of labor mobility and network, the vast majority of rural labor flow in a state of disorder. The Beijing and Tianjing in the two cities city access to set up a high threshold for the reasonable configuration of low-end labor force, but also hinder the regional urbanization. The capital, Beijing has the most superior education resources in China, one of the most abundant human resources, the rich resources of science and technology, these resources should become the engine of the regional economic development, the spillover effect of the Beijing and Tianjing resources far not reached the level of its [11]. By the regional barrier of administrative power not only damage the overall regional interests and long-term interests, also detrimental to the healthy development of the Beijing and Tianjing urbanization. 


\section{The gap between rich and poor urban and rural residents is too large The urban and rural residents income gap is too large}

Our country special long-term development model, the urban and rural dual structure in urban and rural income gap is widening. City occupies the absolute superiority in social resources possession, the countryside is in absolute inferiority in terms of social resources possession. This kind of dual economic structure in Beijing-Tianjing-Hebei region is especially prominent. Within the province (municipality directly under the central government), urban per capita disposable income per capita net income of rural residents is 2.4 times the left and right sides. Comparison between provinces (municipalities directly under the central government), the Beijing urban residents per capita disposable income is 4.9 times per capita net income of rural residents in Hebei province.

\section{Tich cities and poor rural co-exist}

Long-term pattern of urban and rural development, and has formed the prosperity of the modern city and the surrounding backward and poor rural coexist, in the territory of $100 \mathrm{~km}$ border with Beijing and Tianjing Hebei a total of 32 counties and accounted for $44 \%$ of the total number of counties (districts) there. County area of 8.3 square kilometers, accounting for $63.3 \%$ of the total area in the region. A population of 10.63 million people, accounting for $29.2 \%$ of the total population in the region. Funding 3798 of them, the poor of 2.726 million [12]. Area of the living standards of the so big gap between urban and rural residents, and lack of necessary resources to support the urban development and the market space, directly restrict the coordinated development of regional economy as a whole and power to ascend.

\section{The ecological environment is seriously deteriorated The serious shortage of water resources}

Rapid population growth and industrialization, leading to a rapidly increasing consumption of water resources. Beijing-Tianjing-Hebei regional water requirement of less than 6 billion cubic meters in the 6 billion s, the rapid increase to 24 billion cubic meters in 2008, the average annual growth of 300 million cubic meters. 30 billion cubic meters of water quantity in the $1950 \mathrm{~s}$ during the same period fell to 15.5 billion cubic meters at the beginning of the 21st century, almost cut in half. Water consumption is beyond the carrying capacity of the natural environment, self-purification ability and resilience, lead to the serious shortage of water resources of the Beijing-Tianjing-Hebei region. Area of less than 300 cubic meters per year, per capita water resources is $1 / 7$ of the national average; At the same time, due to excessive exploitation of groundwater, in recent 10 years on average groundwater depth decreased from 11.9 meters to 11.9 meters, the annual average fell 1.1 meters [13]. Countries have to make the south-north water diversion project construction, and is used to alleviate the pressure of the Beijing-Tianjing-Hebei region water resources shortage.

\section{Serious environmental pollution}

Beijing-Tianjing-Hebei ecological environment quality in general is on the decline, with the center of the city environment pollution serious, air pollution, ecological destruction intensified the trend has not been effective control. Beijing-Tianjing-Hebei region is town populated areas, is also the most prominent regional haze problem worldwide. Area of the industrialization, urbanization and motorization associated with changes in the atmospheric environment in north China, formed the coal, motor vehicle, symbiotic industrial emissions of pollutants. Beijing-Tianjing-Hebei regional air pollution along the taihang mountain area before extension, high pollution, energy intensive industries, the environmental quality of the Beijing-Tianjing-Hebei region has caused great negative impact. PM2.5 monitoring data 
released by the national 945 monitoring stations, air pollution standard frequency BeijingTianjing-Hebei region the highest, the most concentrated area of pollution in handan, xingtai, etc. At the same time, the area is water pollution in our country, rural non-point source pollution and urban environmental pollution the most serious region [14]. Surface water pollution, especially through the city of organic pollution is more serious. Lake eutrophication problem is outstanding, by dot or planar pollution of groundwater, water level continues to decline, the continuous growth of the population and water pollution, intensified the contradiction between supply and demand of water resources.

\section{BEIJING-TIANJING-HEBEI URBANIZATION COORDINATED DEVELOPMENT COUNTERMEASURE}

\section{Overall planning}

\section{The overall planning of regional urbanization}

Is a set up relevant institutions, urbanization as a whole the Beijing-Tianjing-Hebei region. Situation of three provinces and cities each other space is not conducive to the coordinated development of regional urbanization of the Beijing-Tianjing-Hebei region, it is necessary to set up relevant institutions, coordinate the Beijing-Tianjing-Hebei region urbanization. On the basis of three provinces and related departments, set up a coordinating committee, according to the principle of complementary advantages, mutual sharing of urbanization as a whole the Beijing-Tianjing-Hebei region. Preparation of Beijing-Tianjing-Hebei region urbanization development plan, clear the city function orientation, industrial division layout, infrastructure construction, ecological environment management, integrated traffic system construction goal and task, find out specific measures to realize the coordinated development of region.

\section{The coordinated development of regional urbanization policies}

Regional urbanization must have relevant policy guidance. Shall, according to the regional development present situation and the economic development needs, starting from the concept of greater Beijing, in order to promote regional economic development as the goal, aiming at the coordinated development of urbanization, unified arrangement of BeijingTianjing-Hebei region urbanization process. Adjustment of urban spatial layout, optimize the urban structure, promoting collaboration, improve the level of urban agglomeration integration, improve the level of the comprehensive carrying capacity of cities and the connotation development.

Speed up the construction of urban development in coastal region, Tianjing binhai new area development and opening policy to the coastal port caofeidian, huanghua in Hebei, vigorously develop the coastal port cities, to develop the port economy, lead to the coastal areas of the Beijing and Tianjing population transfer, ease the pressure of population to gather in Beijing area.

\section{As a whole process of regional integration}

3.1.3.1 Is the overall regional industry layout. Beijing-Tianjing-Hebei region of the three provinces and cities in all aspects of social and economic, has a strong complementarity. For a long time, due to the influence of various factors, the lack of unified regional planning and Beijing-Tianjing-Hebei region industry layout, lead to social and economic aspects of coordination is poorer, restricted the rapid development of the region's economy. As a whole the area industry layout, which is beneficial to promote the development of the BeijingTianjing-Hebei region urbanization, is helpful to promote the healthy development of the Beijing-Tianjing-Hebei region economy. 
3.1.3.2 It is to accelerate the market integration process. Beijing-Tianjing-Hebei region economy development, need Beijing-Tianjing-Hebei area market integration support. Take powerful measures to break institutional barriers that affect the regional market integration, promote the production elements such as capital, technology and talent to the free flow and optimized, the development of various elements in free flow and optimized allocation in a region.

3.1.3.3 It is to build regional transportation network. Beijing-Tianjing-Hebei region should be based on urban agglomeration development of urbanization, urban agglomeration coordinated development to have smooth and convenient traffic network support. To build the regional modern traffic network system as a breakthrough point, to speed up building a quick, convenient and efficient comprehensive transport network connectivity, provide transportation network support for the Beijing-Tianjing-Hebei region urbanization.

To speed up the integration process of public service, provide public services support for the coordinated development of Beijing-Tianjin-Hebei urban agglomeration.

\section{To strengthen cooperation}

\section{Strengthen environmental cooperation}

3.2.1.1 Is to strengthen water resources protection. As the coordinated development of urbanization in the periphery of the Beijing-Tianjing-Hebei region, in Hebei province is an important ecological barrier of the Beijing and Tianjing and water source. In recent years, the press, certain achievements have been made in the aspects of sustainable utilization of water resources, but the regional water resources issues has not been well solved. Of Zhangjiakou chengde two cities of Beijing-Tianjing upstream of the ecological environment and water environment problems, directly affect the Beijing-Tianjing water security. Will strengthen the protection of water source of Beijing and Tianjing, Zhangjiakou and Chengde areas built national clean energy base, ecological tourism economic zone, the Beijing-Tianjing ecological garden and leisure tourism base.

3.2.1.2 The second is to strengthen environmental pollution control. After years of governance, environment pollution is very serious, not only influence to the construction and development of these two cities, but also affect the environment of Beijing and Tianjing two city image, capital of security and affect the Beijing-Tianjing-Hebei regional overall development. To strengthen the cooperation of pollution control of the Beijing-Tianjing-Hebei region, give full play to the Beijing and Tianjing technology and talent advantages, promote environmental pollution control of the Beijing-Tianjing-Hebei region, is conducive to improve the level of Beijing-Tianjing-Hebei environmental governance. Increase the intensity of ring ecological environment construction of the Beijing and Tianjing, for the Beijing-Tianjing-Hebei urban coordinated development to provide a good ecological environment.

3.2.1.3 Is to strengthen environmental protection coordination. Ecological environment protection is a long-term and arduous task, hard to finish just by one or two areas. BeijingTianjing-Hebei region ecological environment protection task is very heavy, strengthen ecological environmental protection coordination, joint efforts, the three provinces and cities to complete ecological environmental protection tasks. Strengthening the cooperation of the ecological environment protection, in has been started, on the basis of cooperation mechanism for the control of air pollution, building perfect protection, water conservation, water environmental governance, the use of clean energy cooperation mechanism. As the 
coordinated development of urbanization in the periphery of the Beijing-Tianjing-Hebei region, in Hebei province is an important source of supply of Beijing and Tianjing water resources and ecological barrier, is Beijing and Tianjing security barrier. [15] the ecological environment in Hebei province has very important influence on the development of Beijing and Tianjing, to strengthen the construction of ecological environment in Hebei province and the construction of the ecological and environmental protection, led to the development of Tianjing a good ecological environment.

3.2.1.4 Is to focus on expanding environmental capacity and ecological space, the BeijingTianjing population has reached saturation, land resources, water resources and the energy demand considerably more than Beijing and Tianjing environmental capacity and capacity. Administrative division and geographical restrictions, has affected the population to the Beijing and Tianjing Hebei evacuation, lead to the population of Beijing and Tianjing mounting pressure, ecological space is very nervous, the ecological environment worsening. Establish regional resource sharing mechanism, the rational allocation of resources, expanding environmental capacity and ecological space, eliminate the influence of regional resource sharing all kinds of barriers, promote the healthy development of the Beijing-Tianjing-Hebei region.

\section{Strengthen scientific and technological cooperation}

Only the scientific and technological achievements into productivity, to promote economic development, and to promote the benign development of the scientific research. Due to regional separation and division space, lack of experimental base and achievements transformation base, resulting in a large number of scientific and technological achievements is difficult to translate into actual productivity, impact on the development of economy and the benign development of scientific research.

Beijing and Tianjing has strong scientific research strength and a large number of scientific research achievements, but the lack of high and new technology pilot and achievements transformation base. In Hebei province has a large number of high-tech park and achievements transformation base, and a large number of high quality, low cost of human resources, to Beijing and Tianjing high and new technology to provide experimental base, as Beijing and Tianjing and the transformation of the transformation of high-tech achievements provide talents, promote the Beijing and Tianjing high-tech achievements into realistic productivity as soon as possible. Beijing-shijiazhuang corridor zone of collaborative development project, make full use of high-tech achievements of Beijing and Tianjing, help the upgrading of industrial structure of Hebei region, the maximum compression polluting, energy intensive industries.

Advice led by central, Beijing-Tianjing-Hebei departments of science and technology cooperation coordination group, is responsible for the Beijing-Tianjing-Hebei urban coordinated development of science and technology cooperation. For the coordinated development of Beijing-Tianjing-Hebei urban transformation of scientific and technological cooperation and scientific and technological achievements of policies and measures to encourage the Beijing and Tianjing in Hebei province high-tech achievements and new technology, make full use of high and new technology industrial park of Hebei province and science and technology achievements transformation base, transformation of scientific and technological achievements into economic benefits. Strengthen the coordinated development of Beijing-Tianjing-Hebei urban science and technology cooperation, speed up the transformation of scientific and technological achievements, both scientific research 
departments can bring economic benefits, promote the healthy development of the scientific research work, can also through scientific research achievements transformation and the promotion and application of new and high technology, promote the coordinated development of urbanization of the Beijing-Tianjing-Hebei region.

\section{Strengthen the economic development and cooperation}

Strengthening economic cooperation is the key to promote the healthy development of regional urbanization. Economy is the foundation for the development of the city, the city is the result of economic development. Regional economic development level decide the region urbanization development level, regional level of urbanization plays an important role on the regional economic development. Beijing-Tianjing-Hebei economic zone as an important economic region in northern China, the development of its economy to the north economic development plays an important role. Due to the effect of administrative division, fragmented regional economic construction, lead to repetitive construction and disorderly competition. To strengthen the coordination of regional economic development of the Beijing-Tianjing-Hebei region, promote the human resources, material resources and information resources utility maximization, is helpful to promote the healthy development of the Beijing-Tianjing-Hebei region urbanization.

\section{Strengthening the cooperation key projects}

The coordinated development of Beijing-Tianjing-Hebei urban have a large number of key construction projects, Beijing has a number of national projects, Hebei, Tianjing Binhai new area construction projects has the Caofeidian port and industrial park construction projects, all need a lot of money and high quality talents. Establish key project cooperation mechanisms, make full use of Beijing-Tianjing-Hebei capital and talent advantages, provide funds for the construction of key projects, technology and personnel support, promote the construction of key projects. To strengthen the construction of key projects of cooperation and is conducive to give full play to the overall advantages of the Beijing-Tianjing-Hebei region, is advantageous to the construction of key projects of high standard, high quality, promote the coordinated development of Beijing-Tianjing-Hebei urbanization.

The coordinated development of Beijing-Tianjing-Hebei urban agglomeration, we must stick to the overall planning, adhere to reform and innovation. Adhere to the principle of regional advantages complementary and win-win cooperation. According to the construction of BeijingTianjing-Hebei urban agglomeration as the carrier, in order to optimize the ecological environment as the goal, overall regional division of labor and industry layout, adjust the economic structure and spatial structure, the construction of regional transportation network system, construct the system of the coordinated development of Beijing-Tianjing-Hebei longacting mechanism. Promote regional industrial upgrading, to speed up the integration process of public service, build the target direction and measures of the Beijing-Tianjing-Hebei region, complementary advantages, mutual benefit and win-win results of new pattern of coordinated development.

\section{References}

1. to break "a mu 3 distribute land" xi give me at seven o 'clock on the coordinated development of BeijingTianjing-Hebei requirements, 2014-02-27, xinhua net

2. Xi jinping: the coordinated development of Beijing-Tianjing-Hebei is a major national strategy, China website, china.com.cn, 2014-02-28 
3. of the People's Republic of China, the national bureau of statistics: "China statistical yearbook 2013", China statistical publishing house, 2013

4. the census office of the state council, the national bureau of statistics of population and employment statistics division, China's sixth census data in 2010 (1-4), China statistical publishing house, 2012

5. of the People's Republic of China, the national bureau of statistics: "China statistical yearbook 2013", China statistical publishing house, 2013

6. people's government of Hebei province, Hebei economy yearbook 2011 [M], China statistical publishing house, 2011

7. people's government of Hebei province, Hebei economy yearbook 2013 [M], China statistical publishing house, 2013

8. Lv hongping, Yang hui, small towns in Hebei province sustainable development strategy research [J], journal of Hebei university adult education college, 2007, 3

9. Wang Wenlu, a line of double-box under the framework of urbanization in Hebei province [M], China social sciences press, 2005, 9

10. zhang chengcong, Hebei coastal urban agglomeration development research [J], journal of Hebei normal university (philosophy club edition) 2008, 9

11. zhang chengcong, integration of human resources in Beijing, Tianjing and Hebei metropolis circle study [J], population and market analysis, 2006

12. of the People's Republic of China, the national bureau of statistics: "China statistical yearbook 2012", China statistical publishing house, 2012

13. Beijing-Tianjing-Hebei, baidu encyclopedia, http://baike.baidu.com/link? Url = pN3Uj83klfH3MrfpfevBy003TG2aDp-QxbUavSZ5FfTdS8PHjB0fEooYdGW10qkWvnWV7WIZsqNmolkyBAejK \# 5

14. of the people's government of Hebei province urbanization development in Hebei province "twelfth fiveyear" plan [D], the network of the people's government of Hebei province, 2011, 11 